\title{
Simplified DSP-based Signal-Signal Beat Interference Mitigation Technique for Direct Detection OFDM
}

\author{
Zhe Li, Student Member, IEEE, M. Sezer Erk1lınç, Student Member, IEEE, Rachid Bouziane, Benn C. Thomsen, \\ Member, IEEE, Polina Bayvel, Fellow, IEEE, Robert I. Killey, Member, IEEE
}

\begin{abstract}
Short- and medium-haul links in inter-data center, access, and metro networks require cost-effective direct-detection (DD) wavelength division multiplexing (WDM) transceivers offering energy efficiency, high information spectral density (ISD) and dispersion tolerance. Single-sideband orthogonal frequency division multiplexing (SSB-OFDM) with direct detection is a potential solution; however, it suffers a penalty from signal-signal beat interference (SSBI) caused by the square-law photodetection. In this paper, a novel DSP-based SSBI mitigation technique, with lower complexity than previously proposed methods, is proposed and assessed through numerical simulations for the first time. The performance improvement is quantified by simulations of $9 \times 112$ Gb/s 16-QAM SSB-OFDM signal with a net optical ISD of 2.1 (b/s)/Hz. The performance is shown to be similar to that of the more complex receiver-based iterative SSBI compensation technique. Simulations predict an $8.7 \mathrm{~dB}$ reduction in the required OSNR at the 7\% overhead HD-FEC threshold, and increases of up to $100 \%$ in maximum reach over uncompensated standard singlemode fibre (SSMF) using the proposed simplified SSBI compensation technique.
\end{abstract}

Index Terms-Optical communication, spectrally efficient wavelength division multiplexing, signal-signal beat interference cancellation, direct detection, subcarrier modulation, orthogonal frequency division multiplexing, electronic pre-distortion.

\section{INTRODUCTION}

$\mathrm{T}$ HERE is a rapidly increasing demand for capacity in metro, access and inter-data center networks due to data intensive services such as video-on-demand, cloud computing and the internet of things (IoT) [1-3]. To meet this demand, the key requirements for the optical communication links/networks are the cost and energy efficiency, high information spectral density (ISD) and tolerance to noise and fiber impairments [4, 5]. Recently, service providers have started to build $100 \mathrm{~Gb} / \mathrm{s}$ metro optical solutions using direct-detection (DD) technology [6], mainly because the use of single-polarization directdetection WDM systems is a promising low-cost solution to achieve these aims [7]. Compared to coherent detection, which offers the highest spectral efficiency but requires complex

Manuscript received XX, 2015. This work has been supported by the EU ERA-NET+ project, UK EPSRC UNLOC EP/J017582/1 project, EU FP7 ASTRON project and Semtech Corporation. transceiver designs [8-12], DD transceivers use a low-cost, simple receiver structure, which may be favorable for short- and medium- haul applications.

In DD applications, high ISD can be achieved using subcarrier modulation (SCM), with the amplitude and phase of the subcarrier(s) being recovered at the receiver through beating with the optical carrier. There are two SCM approaches being studied for DD systems: optical orthogonal frequency division multiplexing (OFDM) [13] and Nyquist pulse-shaped subcarrier modulation (Nyquist-SCM) [14-17].

Nonlinear distortion arises in DD SCM schemes (both OFDM and Nyquist-SCM) from the signal-signal beating during the square-law detection, generating unwanted mixing products which interfere with the desired signal-carrier beat terms. This effect is known as signal-signal beat interference (SSBI) and causes a significant degradation in receiver sensitivity [18]. Therefore, it is necessary to develop effective techniques to cancel the SSBI, and consequently, improve the performance of DD SCM transmission systems.

Recently, a number of SSBI compensation techniques have been investigated for both OFDM and Nyquist-SCM systems [19-34], including the use of an optical balanced receiver [19, 21], iterative digital pre-distortion [22-24] or iterative digital post-compensation [25-28] schemes. Although the optical scheme provides superior performance, it has the drawback of the significantly increased complexity of the receiver optical hardware, requiring a balanced receiver which includes two single-ended photodiodes and a very narrow optical filter $(<1$ $\mathrm{GHz}$ ) to separate the optical carrier from the transmitted signal. The use of iterative digital pre-distortion leads to an increased peak-to-average-power ratio (PAPR) and imperfect SSBI cancellation introduced by extra beating products after squarelaw detection [28]. The iterative post-compensation scheme proposed in [25] compensates the SSBI with low receiver optical hardware complexity, but the digital hardware complexity of the receiver is high due to the multiple iterations required.

A comparison between the iterative SSBI pre-distortion and

The authors are with the Optical Networks Group, Department of Electronic \& Electrical Engineering at University College London, WC1E 7JE, U.K. (email: zhe.li@ee.ucl.ac.uk; m.erkilinc@ee.ucl.ac.uk; r.bouziane@ucl.ac.uk; b.thomsen@ucl.ac.uk; p.bayvel@ucl.ac.uk; r.killey@ucl.ac.uk). 
post-compensation has been presented in [34]. The results indicate that the two methods have the same computational complexity due to the requirement for the same number of iterations, and iterative SSBI post-compensation exhibits a better SSBI compensation performance.

In this paper, we demonstrate a novel SSBI compensation technique, which combines the concepts of pre-distortion and post-compensation using a single-ended photodiode. This technique offers very good performance, and at the same time avoids the need for multiple iterations of the demodulation DSP at the receiver, while maintaining a simple transceiver hardware configuration. While the technique is suitable for both OFDM and Nyquist-SCM, we demonstrate it in this paper with OFDM in simulations of a $9 \times 112 \mathrm{~Gb} / \mathrm{s}$ WDM single-sideband (SSB) 16-QAM OFDM system on a $50-\mathrm{GHz}$ grid. The results presented include the reductions in the required optical signalto-noise ratio (OSNR) and in the bit error ratio (BER) following transmission over uncompensated standard single-mode fibre (SSMF). Additionally, the compensation performance is compared with the receiver-based iterative SSBI compensation technique [25] and shown to be very similar.

\section{PRinciple OF Operations}

A schematic of the direct-detection WDM system architecture is shown in Fig. 1. The principle and mathematical models of two effective digital SSBI compensation methods are presented in this section: the receiver-based iterative SSBI estimation and cancellation and the proposed simplified SSBI mitigation technique, respectively.

\section{A. Receiver-based iterative SSBI compensation technique}

An iterative SSBI cancellation technique at the receiver has been proposed and successfully applied to DD virtual SSB optical OFDM (VSSB-OOFDM) systems [25], and this technique has been refined and extended to the DD SSB Nyquist-SCM technique [27, 28]. The steps of the technique are described in [25-28].

The SSB subcarrier modulated signal (OFDM or Nyquist$\mathrm{SCM})$ field, $E_{0}(n)$, is generated, where $n$ is the discrete time index. Following this, chromatic dispersion pre-compensation (CDP) by linear convolution with the inverse of the channel response [35] and pre-emphasis can be performed to mitigate the accumulated dispersion after transmission and low-pass filtering effects in the transmitter respectively. During optical modulation, the real-valued optical carrier, $E_{\text {carrier }}$ is added.

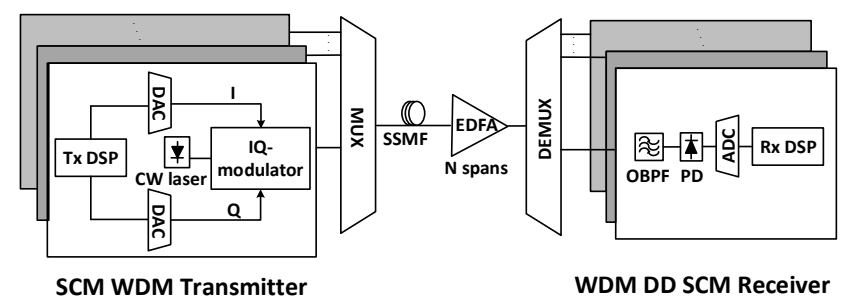

Fig. 1: System architecture for DD WDM transmission system. DAC: digital-to-analogue converter; OBPF: optical band-pass filter; PD: photodetector; ADC: analogue-to-digital converter.
After transmission and square-law detection, the normalized detected signal $V_{D D}(n)$ can be written as:

$$
\begin{aligned}
V_{D D}(n) & =\left|E_{\text {carrier }}+E_{0}(n)\right|^{2} \\
& =E_{\text {carrier }}^{2}+2 \operatorname{Re}\left[E_{\text {carrier }} \cdot E_{0}(n)\right]+\left|E_{0}(n)\right|^{2}
\end{aligned}
$$

where $R e[x]$ signifies the real part of $x$. In the RHS of this equation, the first term is simply the direct current (DC) component, the second term is the desired carrier-signal beating products, and the third is the signal-signal beating products. Within the iterative estimation and cancellation process at the receiver, after demodulation and symbol decisions, modulation DSP is used to generate a digital representation of the ideal single-sideband SCM signal (without the optical carrier), $E_{0}{ }^{\prime}(n)$, and an approximation of the waveform of the signal-signal beating products is obtained by implementing the square-law detection process:

$V_{\text {construct }}(n)=\left|E_{0}^{\prime}(n)\right|^{2}$

where $V_{\text {construct }}(n)$ is the reconstructed signal-signal beating products. This is then subtracted from the stored received signal waveform, partially cancelling the distortion due to signalsignal beating. Since the symbol decisions, and, consequently, $V_{\text {construct }}$ are not accurate for all the symbols, multiple iterations of the demodulation/modulation and SSBI cancellation are required until no further significant gains are observed. When the performance improvement saturates, $E_{0}^{\prime}(n) \approx E_{0}(n)$, and the compensated signal, $V_{\text {compensate }}(n)$ is written as:

$$
\begin{aligned}
V_{\text {compensate }}(n) & =V_{D D}(n)-V_{\text {construct }}(n) \\
& \approx E_{\text {carrier }}^{2}+2 \operatorname{Re}\left[E_{\text {carrier }} \cdot E_{0}(n)\right]
\end{aligned}
$$

Therefore, the compensated signal only contains the DC and the desired carrier-signal beating terms, and the nonlinear distortion caused by square-law detection is reduced. Typically, three or four iterations are required to reach the fundamental limit of the compensation performance, which results in significant DSP complexity [25-28].

\section{B. Simplified SSBI compensation technique}

The simplified SSBI compensation technique that we propose here for the first time has two steps: simple transmitterbased digital pre-distortion and a receiver-based estimation and cancellation technique. The system performance is improved by the pre-distortion which leads to more accurate decision making

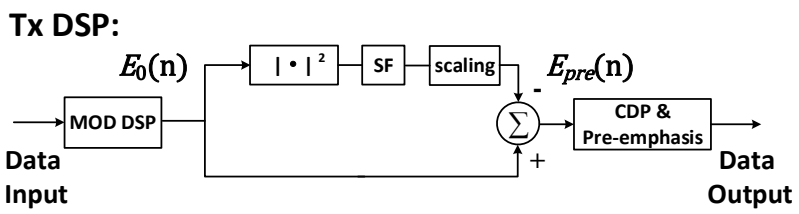

Fig. 2: Transmitter DSP with SSBI pre-distortion, chromatic dispersion pre-compensation (CDP) and pre-emphasis. MOD DSP: single-sideband OFDM or Nyquist-SCM signal generation; SF: sideband filter 
and SSBI estimation at the receiver, thus avoiding the need to perform multiple iterations in the post-compensation DSP.

The principle of the SSBI pre-distortion is shown in Fig. 2. Following the generation of the digital representation of the single-sideband SCM signal by the modulation DSP, a digital representation of the signal-signal beating products is calculated, which is subtracted from the original signal to predistort the signal. This pre-distortion technique can be described as follows:

$$
E_{p r e}(n)=E_{0}(n)-\eta \Lambda\left[\left|E_{0}(n)\right|^{2}\right]
$$

where $E_{\text {pre }}(n)$ is the pre-distorted signal, $E_{0}(n)$ is the singlesideband SCM signal after modulation DSP, $\eta$ is the scaling parameter which controls the SSBI pre-distortion effectiveness, $\Lambda[\cdot]$ is the operator describing the effect of the sideband filter and $n$ is the discrete time index. Note that, since the signalsignal beating terms fall within the bandwidth of the original signal, the proposed pre-distortion does not cause any bandwidth increase, and hence does not introduce any additional penalty due to bandwidth limitations. Following this, digital chromatic dispersion precompensation and preemphasis are carried out for dispersion and low-pass filtering compensation respectively.

After electrical-to-optical conversion (with the addition of the optical carrier), transmission over the fiber and, finally, square-law detection, the detected signal $V_{D D}(n)$ can be written as:

$$
\begin{aligned}
& V_{D D}(n)=\left|E_{\text {carrier }}+E_{\text {pre }}(n)\right|^{2} \\
& \quad=E_{\text {carrier }}^{2}+2 \operatorname{Re}\left[E_{\text {carrier }} \cdot E_{0}(n)\right]+\left|E_{0}(n)\right|^{2}+\eta^{2}\left|\Lambda\left[\left|E_{0}(n)\right|^{2}\right]\right|^{2} \\
& -2 \eta \operatorname{Re}\left[E_{0}(n)^{*} \cdot \Lambda\left[\left|E_{0}(n)\right|^{2}\right]\right]-2 \eta \operatorname{Re}\left[E_{\text {carrier }} \cdot \Lambda\left[\left|E_{0}(n)\right|^{2}\right]\right]
\end{aligned}
$$

In this equation, the first term is simply the DC component, the second term is the desired carrier-signal beating products, and the third to the sixth terms represent additional beating products between the signal, the signal-signal beating products and the optical carrier. With the optimum adjustment of the scaling factor, $\eta$, and the optical carrier, $E_{\text {carrier, }}$, at the transmitter, the third term (signal-signal beating products) is partially cancelled by the sixth term. On the other hand, the fourth and fifth terms are relatively low, so the total amount of nonlinear distortion is reduced with respect to that without SSBI pre-distortion.

At the receiver, the SSBI estimation and cancellation technique shown in Fig. 3 is used. The principle is as follows: (a) the detected signal waveform is stored in memory, and then demodulation DSP, including frequency down-conversion, subcarrier demultiplexing and equalization, is carried out; (b) after making symbol decisions, a digital representation of the pre-distorted signal is re-generated and the effect of square-law detection is calculated. For the accurate calculation of the waveforms of the remaining beating terms after SSBI predistortion, the beating terms between the carrier and signalsignal beating products are re-generated and subsequently, subtracted from the detected signal waveform; (c) for synchronization and amplitude scaling, correlation calculations are carried out to determine the correct delay and amplitude scaling of the reconstructed beating terms, which are then subtracted from the stored received signal to eliminate the interfering terms; (d) finally, the compensated signal is demodulated and BER is calculated. In contrast to the previously demonstrated SSBI post-compensation technique $[25,26]$, no training sequence is required in this algorithm, only correlation calculations are sufficient for accurate synchronization.

Since the predistortion at the transmitter partially compensates for the SSBI, multiple iterations of the above procedure are not required to achieve maximum gain. Hence the technique is simpler than the iterative techniques previously proposed.

The principle of the post-compensation technique is mathematically described below:

$$
\begin{aligned}
& \begin{aligned}
V_{\text {construct }}(n) & =\left|E_{0}^{\prime}(n)-\eta \Lambda\left[\left|E_{0}^{\prime}(n)\right|^{2}\right]\right|^{2}-2 \eta \operatorname{Re}\left[E_{\text {carrier }} \cdot \Lambda\left[\left|E_{0}^{\prime}(n)\right|^{2}\right]\right] \\
& =\left|E_{0}^{\prime}(n)\right|^{2}+\eta^{2}\left|\Lambda\left[\left|E_{0}^{\prime}(n)\right|^{2}\right]\right|^{2}-2 \eta \operatorname{Re}\left[E_{0}^{\prime}(n)^{*} \cdot \Lambda\left[\left|E_{0}^{\prime}(n)\right|^{2}\right]\right]
\end{aligned} \\
& -2 \eta \operatorname{Re}\left[E_{\text {carrier }} \cdot \Lambda\left[\left|E_{0}^{\prime}(n)\right|^{2}\right]\right]
\end{aligned}
$$

where $V_{\text {construct }}(n)$ is the reconstructed beating terms, and $E_{0}{ }^{\prime}(n)$ is the regenerated SCM signal based on the symbol decisions. After subtraction from the stored signal waveform, assuming $E_{0}^{\prime}(n) \approx E_{0}(n)$, the compensated signal, $V_{\text {compensate }}(n)$ can be given as:

\section{Rx DSP:}

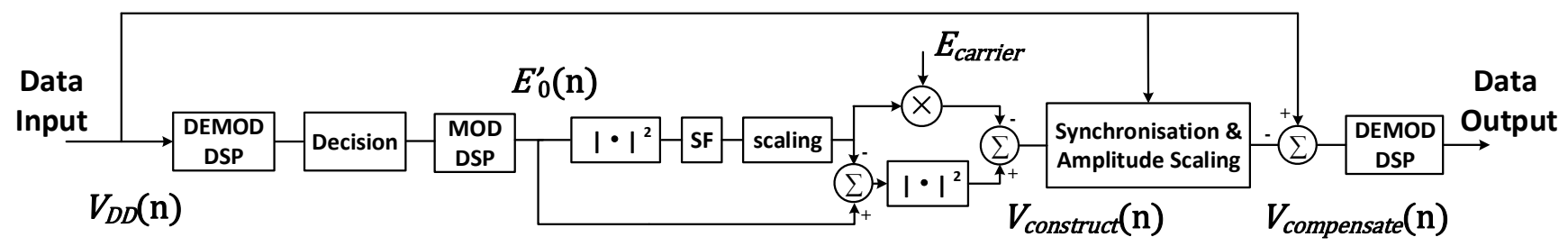

Fig. 3: Receiver DSP with SSBI post-compensation. MOD \& DEMOD DSP: single-sideband OFDM or Nyquist-SCM signal generation \& demodulation; SF: sideband filter; 


$$
\begin{aligned}
V_{\text {compensate }}(n) & =V_{D D}(n)-V_{\text {construct }}(n) \\
& \approx E_{\text {carrier }}^{2}+2 \operatorname{Re}\left[E_{\text {carrier }} \cdot E_{0}(n)\right] .
\end{aligned}
$$

This is the same as equation (3), from which it can be seen that the SSBI is compensated.

\section{Simulation Methodology}

The performance of the proposed SSBI compensation technique was tested in simulations of a WDM DD single-sideband OFDM system, and was compared with the receiver-based iterative SSBI estimation and cancellation technique. The signal generation, transmission and detection were modeled using MATLAB. The modulation DSP (MOD DSP in Figs. 2 and 3) was carried out as follows: the input bit sequences were mapped to symbols to generate a conventional 16-QAM signal. The symbol rate, $f_{s}$, was set to $28 \mathrm{GBaud}$, corresponding to a bit rate of $112 \mathrm{~Gb} / \mathrm{s}$. Subsequently, the single-sideband OFDM signal was generated. 256 data subcarriers were multiplexed using a 512-point inverse fast Fourier transform (IFFT). In order to avoid inter-symbol interference (ISI) due to delays introduced by electrical and optical filters in the system, a $2 \%$ cyclic prefix (CP) was added.

For mitigation of SSBI, chromatic dispersion and low-pass filtering effects, SSBI pre-distortion, CDP and pre-emphasis were carried out following the modulation DSP in the transmitter. To further optimize the system performance, symmetric clipping was performed and the signal PAPR was reduced to $8.8 \mathrm{~dB}$ after clipping. The I- and Q-components of the SSB-OFDM signal were generated using a pair of digitalto-analogue converters (DACs). The resolution of the DACs was assumed to be 5 bits and the transmitter electrical bandwidth was $30 \mathrm{GHz}$, modelled with a $5^{\text {th }}$ order Bessel lowpass filter. The spectrum of the digital SSB-OFDM signal is shown in Fig. 4(a). The optical carrier was added by optimally biasing the IQ-modulator to achieve linear mapping from the electrical to optical domain with the desired carrier-tosignal power ratio (CSPR). All 9 WDM channels, each carrying a $112 \mathrm{~Gb} / \mathrm{s}$ single-sideband 16-QAM OFDM signal encoding four de-correlated $2^{18}$ de Bruijn sequences, with $50-\mathrm{GHz}$ channel spacing, were de-correlated by more than 120 symbols and then multiplexed. The WDM optical spectrum is shown in Fig. 4 (b). Following this, transmission over the fibre link was simulated. It should be mentioned that, in order to maintain the effectiveness of the SSBI pre-distortion, the value of the scaling factor, $\eta$, needs to be chosen based on the CSPR value being used. For simulations described in this paper, the scaling factor was set to 1.0 at BER $>10^{-3}$ (without SSBI compensation) and was adjusted to 0.5 for $\mathrm{BER}<10^{-3}$.

The transmission link considered used uncompensated standard single mode fibre (SSMF), and consisted of multiple fibre spans with the span length $L$, dispersion $D$, fibre loss $\alpha$ and nonlinear coefficient $\gamma$ chosen as $80 \mathrm{~km}, 16.8 \mathrm{ps} /(\mathrm{nm} \cdot \mathrm{km}), 0.2$ $\mathrm{dB} / \mathrm{km}$ and $1.2 \mathrm{~W}^{-1} \mathrm{~km}^{-1}$, respectively. The fibre loss $(16 \mathrm{~dB})$ of each span was compensated by an Erbium-doped fibre amplifier (EDFA) with a noise figure of $5 \mathrm{~dB}$. The transmission was modelled using the symmetric split-step Fourier method

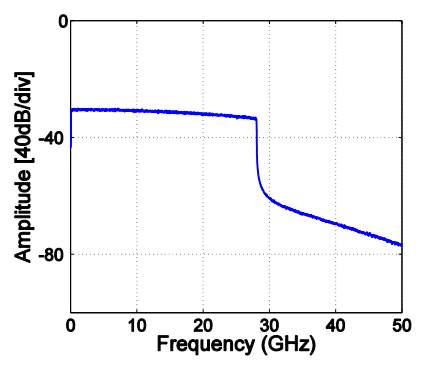

(a)

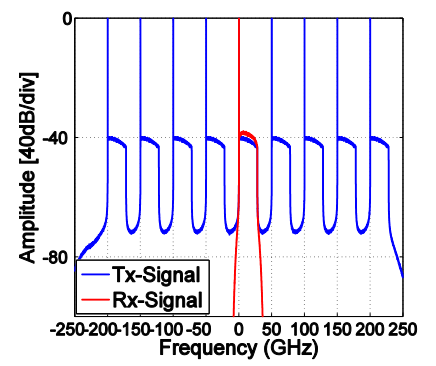

(b)
Fig. 4: (a) Digital 16-QAM SSB-OFDM spectrum (b) WDM 16-QAM SSB-OFDM optical spectra before and after OBPF. Note that frequency scales in the optical spectra are relative to the optical carrier frequency of the central channel.

[36] to solve the nonlinear Schrödinger equation (NLSE), and the step size was empirically chosen as $0.02 \mathrm{~km}$ at a bandwidth of approximately $500 \mathrm{GHz}$.

Following transmission, the channel of interest was demultiplexed by applying a $3^{\text {rd }}$-order super-Gaussian optical band-pass filter (OBPF) with a $3 \mathrm{~dB}$ bandwidth of $30 \mathrm{GHz}$. For all the results described in this paper, the detected channel was the central WDM channel, as shown in Fig. 4(b). The opticalto-electrical conversion was performed by square-law detection using a single-ended photodiode with a $3 \mathrm{~dB}$ bandwidth of 30 $\mathrm{GHz}$ ( $5^{\text {th }}$ order Bessel lowpass filter). The received electrical signal was digitized by a single ADC with a resolution of 5 bits. In the receiver DSP, after normalization, the demodulation DSP (labelled as DEMOD DSP in Fig. 3) consisted of the following steps: serial to parallel conversion, CP removal, 512-points FFT and single-tap frequency domain equalizer to compensate for the phase errors and distortions incurred along the transmission. Block-type channel estimation, utilizing training symbols at the beginning of transmitted sequence, was carried out. The coefficients of the single-tap equalizer were derived by comparing the received training symbols with the transmitted ones [37] and polynomial fit was utilized to the derived coefficients for improved channel estimation. Finally, the received signal was restored by multiplying with the inverse of the estimated channel response. Following this, hard decisions were made on the 16-QAM symbols, the proposed simplified SSBI post-compensation method was applied to compensate the SSBI. Finally, BER (by error counting over $2^{20}$ bits) and error vector magnitude (EVM) [38] were calculated. In order to evaluate the proposed SSBI compensation performance, the previously proposed receiver-based iterative SSBI estimation and cancellation technique was also performed for comparison, with the number of iterations set to four to achieve the optimum performance.

In DD SCM optical communication systems, there is a tradeoff between the SSBI and the signal-ASE beating noise. Signals with lower CSPR suffer from high SSBI, while high CSPR leads to large signal-ASE beating noise penalties. Hence, it is crucial to ensure that the system is operating at the optimum CSPR value. However, after SSBI compensation, the trade-off is changed (due to the lower impact of SSBI), therefore the CSPR value needs to be reduced to the new lower optimum 
value to maximize the performance of SSBI compensation technique. In this paper, for performance optimization, the CSPR used for the system implementing SSBI compensation was reduced by $3 \mathrm{~dB}$ with respect to the value for the uncompensated system. The optimum values of CSPR for systems implementing simplified and receiver-based iterative SSBI compensation methods are similar due to the unchanged tradeoff between the SSBI and ASE-noise beating, despite the differences in the SSBI compensation schemes. Furthermore, although a very strong carrier (corresponding to high CSPR) is applied, the total transmitted optical power is below the Stimulated Brillouin scattering (SBS) threshold [39], and thus, the SBS effect is not considered in the simulations.

\section{RESULTS AND DISCUSSION}

\section{A. Optical Back-to-back Performance}

The optical back-to-back performance evaluation was carried out by performing ASE-noise loading at the receiver to measure the BER versus OSNR. The CSPR was swept from $5 \mathrm{~dB}$ to 13 $\mathrm{dB}$ at each OSNR level to obtain the optimum system performance. The BER was measured and the resultant curves plotted as functions of OSNR for the cases without and with (simplified and receiver-based iterative) SSBI compensation methods (Fig. 5 (a)). It can be seen that the system performance is significantly improved after applying both SSBI compensation techniques. The required OSNR value at the 7\% overhead hard-decision forward error correction (HD-FEC) threshold (assumed to be BER $=3.8 \times 10^{-3}$ ) is found to be 36.6 $\mathrm{dB}$ without SSBI compensation, with an improvement by 8.7 $\mathrm{dB}$ to $27.9 \mathrm{~dB}$ for the case with simplified SSBI compensation, and by $9.2 \mathrm{~dB}$ to $27.4 \mathrm{~dB}$ for the case with receiver-based iterative SSBI compensation. The slight difference $(0.5 \mathrm{~dB})$ between the two compensation methods is mainly due to the additional beating between the signal-signal beat products and ASE-noise.

In order to observe the effect of SSBI compensation, the optical back-to-back signal constellations at a high OSNR value

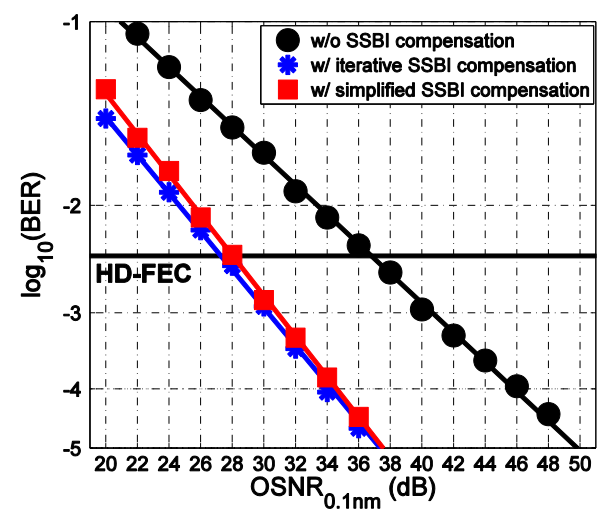

(a)

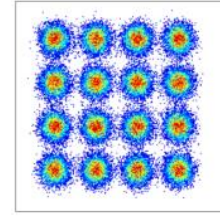

(b)

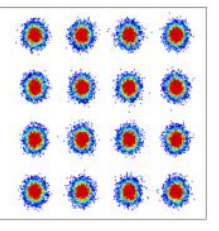

(c)
Fig. 5: (a) BER versus OSNR without and with iterative and simplified SSBI compensations (left). Back-to-back received constellation diagram (right) at the OSNR of $36 \mathrm{~dB}$, (b) without (EVM = 16.9\%) and (c) with $(\mathrm{EVM}=10.0 \%)$ simplified SSBI compensation.

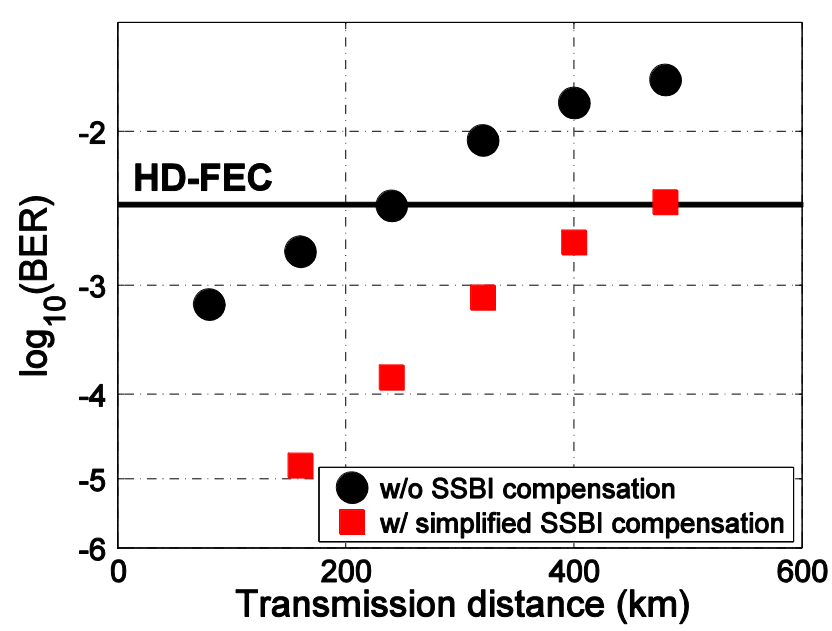

Fig. 6: BER versus transmission distance without and with SSBI compensation.

(36 dB), without and with the simplified SSBI compensation are plotted in Fig. 5 (b) and (c). It can be observed that, after applying SSBI compensation, the compensated constellation is significantly less distorted than the uncompensated one. The error-vector-magnitude (EVM) decreased from $16.9 \%$ to $10.0 \%$ after applying the SSBI compensation. Therefore, in the optical back-to-back case, the proposed simplified SSBI compensation technique can significantly mitigate the impact of SSBI penalty caused by square-law detection, and at the same time has a very similar performance to the receiver-based digital iterative SSBI mitigation technique, but with lower digital hardware complexity.

\section{B. WDM Transmission Performance}

Following the assessment of the back-to-back performance, WDM transmission simulations were carried out for the cases without and with simplified SSBI compensation. Transmission assessments from 1 span $(80 \mathrm{~km})$ to 6 spans $(480 \mathrm{~km})$ of uncompensated SSMF were carried out and the calculated BER

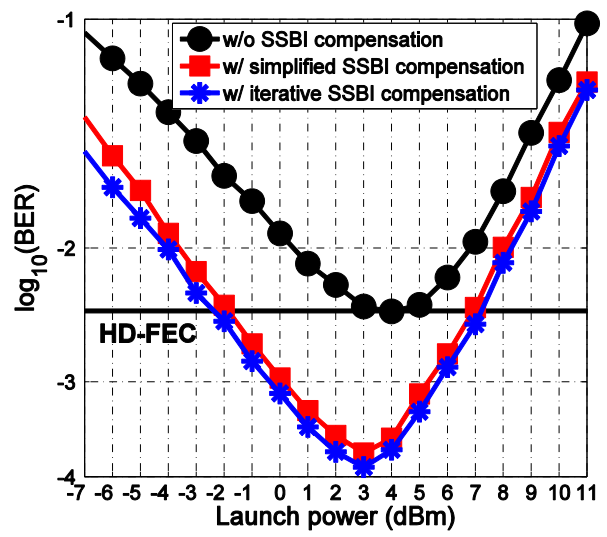

(a)

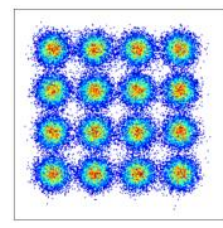

(b)

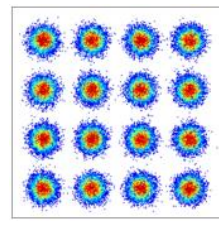

(c)
Fig. 7 (a) BER versus optical launch power at $240 \mathrm{~km}$ WDM transmission without and with iterative and simplified SSBI compensation methods (left). The received constellation diagrams at $240 \mathrm{~km}$ (right), (b) without $(\mathrm{EVM}=17.9 \%)$ and $(\mathrm{c})$ with $(\mathrm{EVM}=12.8 \%)$ simplified SSBI compensation. 


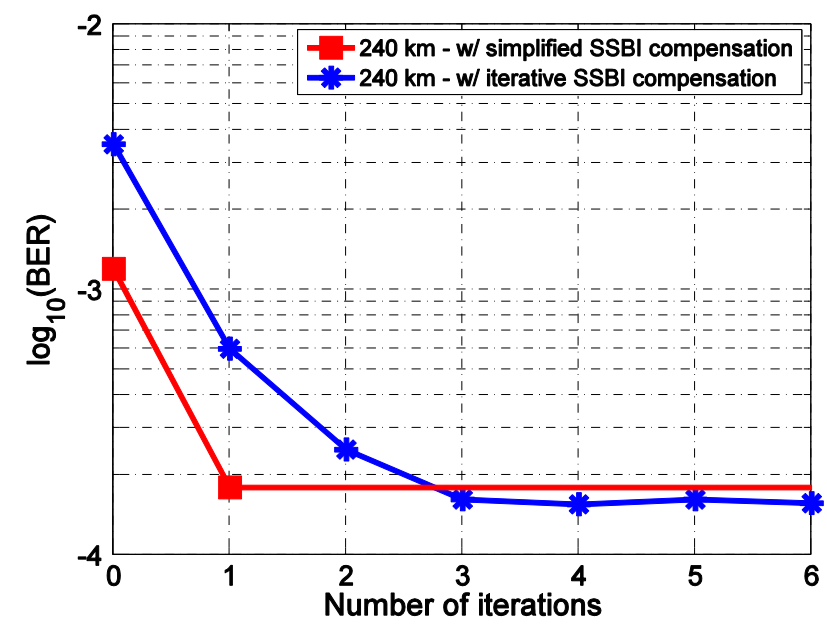

Fig. 8: BER versus the receiver iteration number using the simplified SSBI compensation scheme and iterative compensation at $240 \mathrm{~km}$ transmission distance.

values at the optimum optical launch power per channel were obtained. BER versus transmission distance is shown in Fig. 6. To provide the optimum system performance, the CSPR value was optimized to $16 \mathrm{~dB}$ without and $13 \mathrm{~dB}$ with SSBI compensation at the FEC threshold. It can be observed from the results that the proposed SSBI compensation technique significantly improves the WDM transmission performance at all distances. With this technique, the BER is improved by more than one order of magnitude for distances up to 4 spans (320 $\mathrm{km})$. The achievable transmission distance at the $7 \%$ overhead HD-FEC threshold was extended by $100 \%$ (from $240 \mathrm{~km}$ to 480 $\mathrm{km}$ over uncompensated SSMF) after implementing the proposed SSBI compensation technique. The reduction in gain of SSBI compensation with increasing transmission distance results from penalties due to fibre nonlinearities becoming more significant and dominating the system performance at the longer transmission distances.

For a comparison with the receiver-based iterative SSBI mitigation technique, the system performance at $240 \mathrm{~km}$ transmission distance was evaluated by plotting the BER versus optical launch power per channel (Fig. 7(a)). It can be seen that the simplified SSBI compensation offers almost the same performance as the iterative SSBI mitigation technique. In comparison to the uncompensated case, the optimum optical launch power was reduced by approximately $1 \mathrm{~dB}$. The received constellation diagrams without and with the simplified SSBI mitigation technique are shown in Fig. 7 (b) and (c). The measured EVM value decreased from $17.9 \%$ to $12.8 \%$ after implementing the proposed SSBI cancellation technique. Therefore, the technique is shown to significantly improve the WDM DD SSB-OFDM transmission performances.

Furthermore, the transmission performance of these two SSBI compensation techniques was compared by plotting the BER versus the number of iterations for a transmission distance of $240 \mathrm{~km}$, as shown in Fig. 8. It can be seen that the iterative SSBI mitigation scheme requires multiple (typically 3) iterations at the receiver to fully exploit the SSBI compensation performance. However, the simplified SSBI mitigation scheme offers very similar performance while avoiding the need for multiple iterations.

Further work will be required to assess the effects of electrical bandwidth limitations of the components and nonnegligible DAC/ADC quantization noise on the SSBI compensation scheme in low cost systems.

\section{CONCLUSIONS}

We proposed and assessed, for the first time, a novel signalsignal beat interference (SSBI) cancellation technique with low electronic hardware complexity for WDM direct-detection (DD) single-sideband orthogonal frequency-division multiplexing (SSB-OFDM) systems. This technique combines the use of digital pre-distortion and post-compensation, employing a single-ended photodiode. At the transmitter, a simple pre-distortion method is carried out as the first stage of the SSBI cancellation method to partially compensate the SSBI and reduce the DSP complexity at the receiver. Then, the receiver-based SSBI estimation and cancellation technique further cancels the interfering beating products without the need to carry out multiple iterations. The performance of this technique was tested on a simulated spectrally-efficient (2.1 $(\mathrm{b} / \mathrm{s}) / \mathrm{Hz}) 9 \times 112 \mathrm{~Gb} / \mathrm{s}$ WDM SSB $16-\mathrm{QAM}$ OFDM system on a $50-\mathrm{GHz}$ grid, and was compared with the receiver-based iterative SSBI mitigation technique, which performs well but has higher DSP complexity. The optical back-to-back operation and transmission performance without and with the SSBI mitigation techniques were assessed. It was shown that the proposed simplified SSBI compensation technique has a performance very similar to the receiver-based iterative cancellation technique, and significantly reduces the penalty caused by nonlinear distortion due to square-law detection. The back-to-back operation showed a reduction of $8.7 \mathrm{~dB}$ in the required OSNR at the HD-FEC threshold. Following this, simulations of WDM transmission were performed. Utilizing the proposed SSBI compensation technique, the performance was significantly improved at all distances. The achievable transmission distance was extended by $100 \%$ (from $240 \mathrm{~km}$ to $480 \mathrm{~km}$ over uncompensated SSMF). Furthermore, the optimum optical launch power per channel was reduced by approximately $1 \mathrm{~dB}$. To the best of our knowledge, this is the first proposal and assessment of this simplified SSBI estimation and cancellation technique, which may be favorable for lowcost direct-detection OFDM WDM transceivers offering dispersion tolerance and high information spectral density for short- and medium- haul links in metro, access and inter-data centre, $100 \mathrm{G}$ or $1 \mathrm{~T}$ Ethernet links. The proposed scheme is also suitable for Nyquist-SCM WDM transceivers.

\section{REFERENCES}

[1] Alcatel-Lucent, "Bell labs metro network traffic growth: architecture impact study," Strategic White Paper, 2013.

[2] Cisco, "Cisco visual networking index: forecast and methodology, 20142019," White Paper, 2015.

[3] L. Atzori, A.Lera, and G. Morabito, "The internet of things: a survey," Comput. Networks, vol. 54, no. 15, pp. 2787-2805, 2010.

[4] J.M. Kahn and K.-P. Ho, "Spectral efficiency limits and modulation/detection techniques for DWDM systems," IEEE J. Sel. Topics Quantum Electron., vol. 10, no. 2, pp. 259-272, 2004. 
[5] K.-P. Ho, "Exact evaluation of the capacity for intensity-modulated direct-detection channels with optical amplifier noises," IEEE Photon. Technol. Lett., vol. 17, no. 4, pp. 858-860, 2005.

[6] ADVA, "Efficient 100G transport," 2014.

[7] R.I. Killey, M.S. Erkılınç, Z. Li, S. Pachnicke, H. Griesser, R. Bouziane, B.C. Thomsen, and P. Bayvel, "Spectrally-efficient direct-detection WDM transmission system," in Proc. ICTON, 2015, paper We.B3.2.

[8] S. Chandrasekhar, X. Liu, B. Zhu, and D.W. Peckham, "Transmission of a 1.2-Tb/s 24-carrier no-guard-interval coherent OFDM superchannel over 7200-km of ultra-large-area fiber," in Proc. ECOC, 2009, paper PD2.6.

[9] J.-X. Cai, Y. Sun, H.G. Batshon, M. Mazurczyk, H. Zhang, D.G. Foursa, and A.N. Pilipetskii, " $54 \mathrm{~Tb} / \mathrm{s}$ transmission over 9,150 km with optimized hybrid Raman-EDFA amplification and coded modulation," in Proc. ECOC, 2014, paper PD.3.3.

[10] J. Zhang, J. Yu, Y. Fang, and N. Chi, "High speed all optical Nyquist signal generation and full-band coherent detection," Scientific Report, vol. 4, no. 6156, 2014.

[11] S. Beppu, M. Yoshida, K. Kasai, and M. Nakazawa, "2048 QAM (66 Gbit/s) single-carrier coherent optical transmission over $150 \mathrm{~km}$ with a potential SE of 15.3 bit/s/Hz," in Proc. OFC, 2014, paper W1A.6.

[12] T.J. Xia, S. Gringeri, and M. Tomizawa, "High-capacity optical transport networks," IEEE Commun. Mag., vol. 50, no. 11, pp. 170-178, 2012.

[13] A.J. Lowery, L.B. Du, and J. Armstrong, "Performance of optical OFDM in ultralong-haul WDM lightwave systems," J. Lightw. Technol., vol. 25, no. 1, pp. 131-138, 2007.

[14] A.O. Wiberg, B.-E. Olsson, and P.A. Andrekson, "Single cycle subcarrier modulation," in Proc. OFC, 2009, paper OTuE.1.

[15] J.C. Cartledge and A.S. Karar, " $100 \mathrm{~Gb} / \mathrm{s}$ intensity modulation and direct detection," J. Lightw. Technol., vol. 32, no. 16, pp. 2809-2814, 2014.

[16] M.S. Erkılınç, S. Kilmurray, R. Maher, M. Paskov, R. Bouziane, S. Panchnicke, H. Griesser, B.C. Thomsen, P. Bayvel, and R.I. Killey, "Nyquist-shaped dispersion-precompensated subcarrier modulation with direct detection for spectrally-efficient WDM transmission," Opt. Express, vol. 22, no. 8, pp.9420-9431, 2014.

[17] M.S. Erkılınç, Z. Li, S. Pachnicke, H. Griesser, B.C. Thomsen, P. Bayvel, and R.I. Killey, "Spectrally-efficient WDM Nyquist-pulse-shaped 16QAM subcarrier modulation transmission with direct detection," $J$. Lightw. Technol., vol. 33, no. 15, pp. 3147-3155, 2015.

[18] A.J. Lowery, "Amplified-spontaneous noise limit of optical OFDM lightwave systems," Opt. Express, vol. 16, no. 2, pp. 860-865, 2008.

[19] W.R. Peng, I. Morita, and H. Tanaka, "Enabling high capacity directdetection optical OFDM transmissions using beat interference cancellation receiver," in Proc. ECOC, 2010, paper Tu.4.A.2.

[20] S.A. Nezamalhosseini, L.R. Chen, Q. Zhuge, M. Malekiha, F. Marvasti, and D.V. Plant, "Theoretical and experimental investigation of direct detection optical OFDM transmission using beat interference cancellation receiver," Opt. Express, vol. 21, no. 13, pp. 15237-15246, 2013.

[21] J. Ma, "Simple signal-to-signal beat interference cancellation receiver based on balanced detection for a single-sideband optical OFDM signal with a reduced guard band," Opt. Letters, vol. 38, no. 21, pp. 4335-4338, 2013.

[22] C. Sánchez, B. Ortega, and J. Capmany, "System performance enhancement with pre-distorted OOFDM signal waveforms in IM/DD systems," Opt. Express, vol. 22, no. 6, pp. 7269-7283, 2014.

[23] C. Ju, X. Chen, N. Liu, and L. Wang, "SSII cancellation in 40 Gbps VSBIMDD OFDM system based on symbol pre-distortion," in Proc. ECOC, 2014, paper P.7.9.

[24] N. Liu, C. Ju, and X. Chen, "Nonlinear ISI cancellation in VSSB NyquistSCM system with symbol pre-distortion," Opt. Commun., vol. 338, pp. 492-495, 2015.

[25] W.R. Peng, X. Wu, K. Feng, V.R. Arbab, B. Shamee, J. Yang, L. C. Christen, A. E. Willner, and S. Chi, "Spectrally efficient direct-detected OFDM transmission employing an iterative estimation and cancellation technique," Opt. Express, vol. 17, no. 11, pp. 9099-9111, 2009.

[26] H. Shi, P. Yang, C. Ju, X. Chen, J. Bei, and R. Hui, "SSBI cancellation based on time diversity reception in SSB-DD-OOFDM transmission systems," in Proc. CLEO, 2014, paper JTh2A.14.

[27] Z. Li, M. S. Erkılınç, S. Pachnicke, H. Griesser, B.C. Thomsen, P. Bayvel, and R.I. Killey, "Direct-detection 16-QAM Nyquist-shaped subcarrier modulation with SSBI mitigation," in Proc. ICC, 2015, pp. 5204-5209.

[28] Z. Li, M. S. Erkılınç, S. Pachnicke, H. Griesser, R. Bouziane, B.C. Thomsen, P. Bayvel, and R.I. Killey, "Signal-signal beat interference cancellation in spectrally-efficient WDM direct-detection Nyquist-pulse- shaped 16-QAM subcarrier modulation," Opt. Express, vol. 23, no. 18, pp. 23694-23709, 2015.

[29] X. Zhang, J. Li, and Z. Li, "SSBI cancellation method for IMDD-OFDM system with a single photodiode," in Proc. PIERS, 2014, paper 2719.

[30] A. Li, D. Che, X. Chen, Q. Hu, Y. Wang, and W. Shieh, "61 Gbit/s directdetection optical OFDM based on blockwise signal phase switching with signal-to-signal beat noise cancellation," Opt. Express, vol. 17, no. 11, pp. 9099-9111, 2009.

[31] M. Schuster, S. Randel, C.A. Bunge, S.C.J. Lee, F. Breyer, B. Spinnler, and K. Petermann, "Spectrally efficient compatible single-sideband modulation for OFDM transmission with direct detection," IEEE Photon. Technol. Lett., vol. 20, no. 9, pp. 670-672, 2008.

[32] C.Y. Wong, S. Zhang, L. Liu, T. Wang, Q. Zhang, Y. Fang, S. Deng, G. N. Liu, and X. Xu, "56 Gb/s direct detected single-sideband DMT transmission over 320-km SMF using silicon IQ modulator," in Proc. OFC, 2015, paper Th4A.3.

[33] L. Zhang, T. Zuo, Y. Mao, Q. Zhang, E. Zhou, G.N. Liu, and X. Xu. "Beyond 100-Gb/s transmission over 80-km SMF using direct-detection SSB-DMT at C-band." J. Lightw. Technol., 2015, DOI: 10.1109/JLT.2015.2464091

[34] C. Ju, N. Liu, X. Chen, "Comparison of iteration interference cancellation and symbol predistortion algorithm for direct detection orthogonal frequency division multiplexing passive optical network," Opt. Eng., vol. 54, no. 8, pp. 0861014, 2015.

[35] R.I. Killey, P.M. Watts, V. Mikhailov, M. Glick, and P. Bayvel, "Electronic dispersion compensation by signal predistortion using digital processing and a dual-drive Mach-Zehnder modulator," IEEE Photon. Technol. Lett., vol.17, no. 3, pp. 714-716, 2005.

[36] G.P. Agrawal, Applications of nonlinear fiber optics, 3rd ed., Academic, 2010.

[37] J.-J. van de Beek, O. Edfors, M. Sandell, S.K. Wilson, and P.O. Borjesson, "On channel estimation in OFDM systems," in Proc. VTC, 1995, vol.2, pp. 815-819.

[38] R.A. Shafik, M.S. Rahman, and A.R. Islam, "On the extended relationships among EVM, BER and SNR as performance metrics," in Proc. ICECE, 2006, pp. 408-411.

[39] V.I. Kovalev and R.G. Harrison, "Threshold for stimulated Brillouin scattering in optical fiber," Opt. Express, vol. 15, no. 26, pp. 1762517630, 2007. 